\title{
Mesure de la ressemblance de la faune utile du poirier avec celle de 43 espèces végétales pour optimiser la composition de haies réservoir d'auxiliaires
}

Jean-François Debras*, Marianne Cousin, René Rieux

Inra - UMR Écologie

des invertébrés,

Site Agroparc,

Domaine Saint-Paul,

84914 Avignon Cedex 9,

France

debras@avignon.inra.fr

* Correspondance et tirés à part

Reçu le 12 juin 2001

Accepté le 24 septembre 2001

Fruits, 2002, vol. 57, p. 55-65 (C) 2002 Cirad/EDP Sciences All rights reserved

DOI: $10.1051 /$ fruits:2002006

RESUMEN EsPaÑoL, p. 65

\section{Assessment of the similarity of pear tree beneficial fauna with 43 vegetal species for optimal value of hedgerows as beneficial reservoirs.}

Abstract - Introduction. As part of pest management practices, those concerning orchard management surroundings are an important objective. Until now this management has been done without any test on a rational basis. This study shows how it is possible to deal with this point. Materials and methods. The entomofauna of 44 woody species including pear trees was studied for 10 months in 1998 by monthly sampling of 30 trees per species. The capture of the entomofauna was carried out by the Fauvel's beating method. Results and discussion. Basic research was carried out on the composition and structure of the canopy communities of leafy trees and of an agrocenosis, the pear orchard. The arthropod community of 43 leafy species was observed. Arthropod stocks were specific. Correlation between the stock of pear beneficial arthropods and some of the 43 tree species sampled was clearly demonstrated. Conclusion. The aim of this study was to point out the role of some plant species in integrated pest management (IPM) practices. Results supply criteria allowing supervision of the choice of tree species in the context of agrosystems. The same approach can be used in many other crops for developing an IPM strategy.

France / Pyrus communis / integrated control / insect control / hedging plants / biological control arthropod / environment

\section{Mesure de la ressemblance de la faune utile du poirier avec celle de 43 espèces végétales pour optimiser la composition de haies réservoir d'auxiliaires.}

Résumé - Introduction. Pour la mise en place de mesures à promouvoir dans le cadre d'une protection intégrée, celles concernant un aménagement des abords des cultures deviennent un objectif principal. Cependant, jusqu'à présent, cet aménagement s'est fait sans véritable critère. La présente étude montre comment il est possible de raisonner le choix des espèces végétales. Matériel et méthodes. L'entomofaune de 44 espèces ligneuses dont celle du poirier cultivé a été observée, pour chacune de ces espèces, à raison de 30 arbres par mois, pendant 10 mois de l'année 1998. La collecte de l'entomofaune a été réalisée par la méthode du battage de Fauvel. Résultats et discussion. Des recherches fondamentales ont été conduites sur la composition et la structure des communautés animales hébergées par les feuillus et comparées à celles d'un verger de poiriers. Le peuplement de 43 espèces ligneuses a ainsi été étudié. Après avoir montré la spécificité des peuplements, des corrélations de rang de Spearman entre le peuplement d'auxiliaires des 43 essences et celui du poirier ont été mises en évidence et montrent une ressemblance entre l'entomofaune utile du poirier et celle de certaines essences végétales. Conclusion. Les résultats obtenus permettent de raisonner le choix des essences pouvant servir de réservoir à une entomofaune prédatrice dans le cadre de la protection intégrée du verger de poiriers. Cette méthode est applicable à d'autres types de culture.

France / Pyrus communis / lutte intégrée / lutte anti-insecte / haie / arthropode auxiliaire / environnement 


\section{Introduction}

L'agriculture, basée pendant des siècles sur la polyculture, parfois en cultures associées, n'était souvent destinée qu'à la subsistance d'une famille. En se modernisant (mécanisation, produits phytosanitaires, fertilisants...), elle s'est spécialisée pour obtenir une meilleure rentabilité et s'est orientée de plus en plus vers la monoculture sur des surfaces de plus en plus grandes. Toutes ces techniques ont permis d'augmenter les rendements de façon spectaculaire et de diminuer les prix de revient.

On est donc arrivé à n'avoir qu'une seule variété voire qu'un seul clone sur plusieurs hectares cultivés. Cet écosystème atrophié, s'il paraissait plus rentable, est devenu plus fragile. Qu'une maladie ou un ravageur l'attaque et l'ensemble est menacé. Pour remédier à une telle situation, on a eu recours à la lutte chimique intensive qui induit certains problèmes liés à cette utilisation massive : plus on y a recours, plus on déséquilibre l'écosystème.

L'agrandissement des parcelles, la suppression des haies et du bocage ont accentué cet appauvrissement. À une flore peu diversifiée correspond un écosystème déséquilibré. Cet appauvrissement biologique se traduit par des risques plus élevés de pullulation de ravageurs peu freinés par la faune auxiliaire. La préservation de la biodiversité est un moyen capital pour limiter ces effets pervers. Le mot "biodiversity " inventé par Walter G. Rosen en 1985 a été popularisé par la conférence des Nations unies pour l'environnement et le développement à Rio de Janeiro en 1992. Comme il est difficile, pour des raisons économiques, d'envisager des cultures associées, il faut donc, pour favoriser cette biodiversité, diversifier et aménager l'environnement des cultures [1, 2].

Dans le cadre de la protection intégrée, l'environnement et les haies composites jouent un rôle de réservoir de biodiversité pour renforcer le peuplement des auxiliaires des monocultures adjacentes [3]. Les aménagements créés à cette fin consistent principalement en des plantations de haies composites de feuillus en mélange. Toutefois, cette pratique est restée surtout empirique [4]. Très peu d'études ont été conduites pour identifier le rôle de réservoir d'auxiliaires des essences utilisées [5]. C'est donc sur la base de critères totalement étrangers à un tel objectif que les végétaux à planter sont habituellement choisis : rôle de brisevent, de productions annexes de bois ou de petits fruits... En outre, les risques dus à l'enrichissement éventuel en ravageurs potentiels sont difficiles à prendre en compte.

Tout assortiment nouveau d'espèces végétales aux abords d'une culture représente un risque pour celle-ci. Les interactions sont multiples et complexes. Parmi elles, toutes ne sont pas forcément avantageuses. Même si un effort préalable est entrepris pour éliminer les végétaux susceptibles d'interactions défavorables connues, certaines, difficilement prévisibles, pourraient en effet se révéler préjudiciables pour la culture concernée [6].

Cependant les essences entretiennent entre elles des interactions mesurables en termes de ressemblances et de différences entre leurs communautés. Diverses méthodes de calcul utilisées permettent d'évaluer ainsi des "distances mathématiques " entre les communautés animales hébergées par des essences différentes [7]. Il est donc possible, sur la base de relevés faunistiques, de calculer la "ressemblance " entre la faune d'une essence cultivée et celle de n'importe quelle essence environnementale [8].

Cet exercice effectué séparément sur le cortège des auxiliaires et sur celui des phytophages devient un outil permettant une estimation objective de l'intérêt (ressemblance ou proximité pour les auxiliaires) et du risque (ressemblance ou distance pour les phytophages) que représente la faune d'une essence pour une culture donnée.

Nous disposons donc d'un moyen de repérer les végétaux présentant un intérêt pour une espèce cultivée donnée. La présente étude a permis de dresser parmi 43 espèces ligneuses une liste d'essences végétales dont le peuplement d'auxiliaires est correctement corrélé avec le peuplement en auxiliaire du poirier et dont le peuplement en phytophages ne présente pas 
de similarité avec le peuplement en phytophages du poirier. Le but de cette étude a été de montrer comment il est possible d'identifier des essences végétales susceptibles de fournir des auxiliaires pour une culture donnée ; ces essences pourront entrer dans la composition de haies composites pour lutter contre les ravageurs dans le cadre d'une agriculture extensive soucieuse de l'environnement et des normes de la protection intégrée [9]. Cette méthode pourra s'appliquer à d'autres types de culture.

\section{Matériel et méthodes}

\subsection{Localisation, période d'étude et matériel végétal}

L'étude a été conduite dans la région d'Avignon - Montfavet (département du Vaucluse, France) dans le domaine des plaines et basses collines. Les observations ont concerné le poirier commun et 43 essences ligneuses fréquemment plantées dans les haies ou poussant spontanément dans l'environnement des cultures. Nous avons identifié le rôle joué par ces essences dans une haie (tableau I). Pour chacune des espèces retenues, 30 arbres ont été échantillonnés chaque mois pendant 10 mois soit 13200 arbres ; un total de 123750 arthropodes a été réparti en 742 espèces ou taxons. Les relevés ont été limités aux zones de lisière des cultures, de quelques dizaines à une centaine de mètres de celles-ci, sans pénétrer en profondeur dans les formations spontanées. Pour une meilleure indépendance des relevés et une meilleure couverture de la région d'étude, les essences ont été échantillonnées sur des surfaces assez étendues (plusieurs ha), réparties sur un grand nombre de localités pour éviter d'échantillonner les mêmes arbres d'un relevé à l'autre.

L'échantillonnage de la faune du poirier a été réalisé sur la parcelle expérimentale de l'Inra de Montfavet. Il s'agit d'une parcelle de 0,5 ha de poiriers de la variété "Leclerc " conduite en gobelet. Elle est bordée d'une haie composite plantée depuis une dizaine d'années. La pression chimique a été fortement réduite depuis 1997 ; le calendrier de traitement se réduit à du Delfin (Agrisense-BCS Ltd., Loiret et Haëntes, S.A.) contre le carpocapse.

\subsection{Technique de collecte}

L'échantillonnage a concerné la faune des frondaisons. Il a été pratiqué selon la méthode de Fauvel [10] à l'aide d'une matraque caoutchoutée et d'un entonnoir de $20 \mathrm{~cm}$ de diamètre, muni d'une grille à mailles de $1 \mathrm{~cm}$ et d'un tube collecteur de $30 \mathrm{~mL}$. Le battage a été réalisé à hauteur d'homme, sur des branches portant environ (20 à 30) $\mathrm{dm}^{3}$ de feuillage, à raison d'une seule branche par arbre et de 30 arbres par essence. Les captures correspondant aux 30 battages d'une même essence ont été cumulées dans un même tube collecteur contenant de l'alcool à $70 \%$. Les relevés ont été effectués chaque mois, de janvier à octobre, soit pendant 10 mois de l'année 1998.

\subsection{Identification des insectes}

La composition des récoltes a été déterminée au laboratoire, sous loupe binoculaire. Les identifications ont été conduites, si possible, jusqu'au genre ou à l'espèce mais, pour nombre de diptères et d'hyménoptères, elle se sont limitées à la famille.

\subsection{Traitement des données}

Le traitement des données a porté sur l'analyse de la composition et de la structure des biocénoses, la spécificité des peuplements et la comparaison des peuplements de phytophages des 43 essences ligneuses observées avec celui du poirier ; ces résultats ont été complétés par la recherche de corrélations entre la faune auxiliaire du poirier et celle de ces 43 essences végétales.

\subsubsection{Indice de Sorensen}

Le rang des taxons a été obtenu en considérant les effectifs des peuplements par ordre décroissant, le taxon de rang 1 étant alors attribué à celui qui possédait l'effectif 
le plus important. Pour montrer que les peuplements en arthropodes des essences végétales étaient spécifiques aux essences, nous avons d'abord projeté sur un axe simple en abscisse le rang du taxon dans l'essence considérée et en ordonnée le rang du taxon dans la communauté générale des 43 essences (figure 1). Nous avons ainsi obtenu une mesure de la spécificité des peuplements exprimée par le paramètre $R^{2}$.

Tableau I.

Liste des essences végétales échantillonnées vis-à-vis de l'entomofaune qu'elles hébergent et valeur de l'indice de Sorensen.

\begin{tabular}{|c|c|c|c|}
\hline Nom de l'essence & Nom commun & Rôle de l'espèce & Indice de Sorensen (S) \\
\hline Pyrus communis & Poirier & Arbre fruitier & 1 \\
\hline Cydonia vulgaris & Cognassier & Intercalaire & 0,285 \\
\hline Cotinus coggygria & Sumac & Intercalaire & 0,272 \\
\hline Viburnum lantana & Viorne mancienne & Intercalaire & 0,270 \\
\hline Aria incana & Aria & Intermédiaire & 0,230 \\
\hline Cerasus mahaleb & Laurier cerise & Intermédiaire & 0,230 \\
\hline Salix alba & Saule & Haut jet & 0,230 \\
\hline Sambucus nigra & Sureau & Intermédiaire & 0,222 \\
\hline Ulmus minor & Ormeau champêtre & Haut jet & 0,222 \\
\hline Salix incana & Saule & Haut jet & 0,214 \\
\hline Crataegus monogyna & Aubépine & Intercalaire & 0,214 \\
\hline Prunus spinosa & Prunelier & Bourrage & 0,200 \\
\hline Quercus pubescens & Chêne & Intermédiaire & 0,193 \\
\hline Populus alba & Peuplier blanc & Haut jet & 0,193 \\
\hline Viburnum tinus & Viorne tin & Intercalaire & 0,190 \\
\hline Tilia platyphylla & Tilleul & Haut jet & 0,173 \\
\hline Malus communis & Pommier & Intermédiaire & 0,173 \\
\hline Cerasus laurocerasus & Laurier Amande & Intermédiaire & 0,150 \\
\hline Buxus sempervirens & Buis & Bourrage & 0,148 \\
\hline Amygdalus communis & Amandier & Intermédiaire & 0,142 \\
\hline Quercus ilex & Chêne & Intermédiaire & 0,137 \\
\hline Cornus mas & Cornouiller mâle & Bourrage & 0,111 \\
\hline Aucuba japonica & Aucuba & Intercalaire & 0,105 \\
\hline Pistacia terebinthus & Pistachier & Intermédiaire & 0,100 \\
\hline Bupleurum fruticosum & Buplèvre & Bourrage & 0,100 \\
\hline Cercis siliquastrum & Arbre de judée & Intercalaire & 0,095 \\
\hline Cornus sanguinea & Cornouiller sanguin & Bourrage & 0,095 \\
\hline Prunus persica & Pêcher & Intermédiaire & 0,095 \\
\hline Populus nigra & Peuplier noir & Haut jet & 0,090 \\
\hline Salix cinerea & Saule & Haut jet & 0,083 \\
\hline Hedera helix & Lierre & - & 0,083 \\
\hline Corylus avellana & Noisetier & Intercalaire & 0,083 \\
\hline Fraxinus angustifolia & Frêne & Haut jet & 0,080 \\
\hline Cupressus sempervirens & Cyprès & Intermédiaire & 0,076 \\
\hline Thuja occidentalis & Thuja & Intercalaire & 0,076 \\
\hline Cupressus arizonica & Cyprès bleu & Intermédiaire & 0,074 \\
\hline Pyrus amygdaliformis & Poirier sauvage & Intercalaire & 0,070 \\
\hline Prunus cerasifera & Prunier & Intercalaire & 0,064 \\
\hline Rhamnus alaternus & Alaterne & Bourrage & 0 \\
\hline Ligustrum japonicum & Troènes & Intermédiaire & 0 \\
\hline Ilex aquifolium & Houx & Intercalaire & 0 \\
\hline Photinia serrulata & Photinia & Intermédiaire & 0 \\
\hline Cupressocyparis leylandi & Cyprès Leyland & Intermédiaire & 0 \\
\hline Laurus nobilis & Laurier & Intercalaire & 0 \\
\hline
\end{tabular}


En isolant alors, de ces peuplements, les taxons phytophages, nous avons pu les comparer avec le peuplement en phytophages du poirier par le coefficient de Sorensen : $\mathrm{S}=[2 \mathrm{C} /(\mathrm{A}+\mathrm{B})]$, où $C$ représente le nombre des espèces communes à deux relevés notés " $\mathrm{a}$ " et " $\mathrm{b}$ " dans lesquels $A$ est le nombre total des espèces présentes dans le relevé " a " et $B$ le nombre total des espèces présentes dans le relevé " $b$ "; le paramètre $C$ représente donc le nombre d'espèces présentes deux fois dans l'ensemble des deux relevés comparés et la somme $(A+B)$ représente la totalité des espèces de ces deux relevés. $S$ peut varier de 0 à 1 .

\subsubsection{Corrélation de rang de Spearman}

Les ressemblances du cortège des auxiliaires entre les diverses essences ont ensuite été mesurées par la méthode des corrélations de rang de Spearman de façon à mettre en évidence d'éventuelles similitudes de peuplement entre essences.

Le coefficient de corrélation de rang de Spearman " $r_{s}$ " indique le degré de liaison entre le classement des éléments selon la variable $x$ et le classement des éléments selon la variable $y$. Si $r_{s}=1$, les classements selon $x$ et $y$ se révèlent rigoureusement identiques ; si $r_{s}=-1$, les deux classements sont rigoureusement inverses ; enfin, si $r_{s}=0$, les deux variables sont indépendantes et l'ordre de classement selon $x$ et $y$ devient aléatoire.

Pour calculer le coefficient de corrélation de Spearman, il faut indiquer le rang de chaque élément dans la série croissante de valeurs de $x$ et de $y$, puis calculer la différence de classement $d_{i}$ pour chaque élément de l'échantillon; il est alors possible d'appliquer la formule de Spearman : $r_{s}=$ $\left[\left(1-6 \sum^{\mathrm{n}}{ }_{\mathrm{i}=1} d^{2}\right) / n\left(n^{2}-1\right)\right]$. Pour $n=15^{s}$ et à $5 \%$, le seuil est $r=0,62$.

\section{Résultats et discussion}

\subsection{Analyse globale du peuplement de l'agrosystème par les arthropodes}

L'analyse globale du peuplement a été effectuée sur quatre grands groupes : les

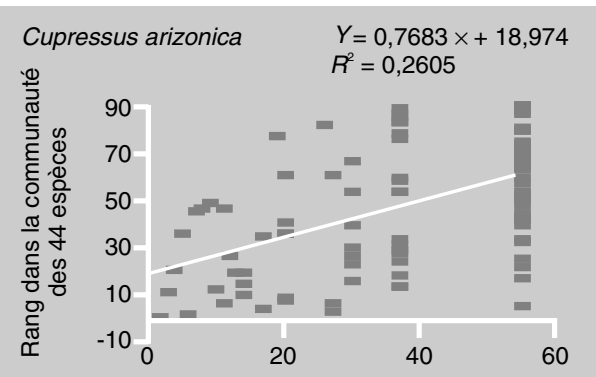

Figure 1.

Mesure de la spécificité du peuplement en arthropodes de quatre espèces de conifères par le calcul du paramètre $R^{2}$. Le rang des taxons a été obtenu en considérant les effectifs des peuplements par ordre décroissant, le taxon de rang 1 étant alors attribué à celui qui possède l'effectif le plus important.

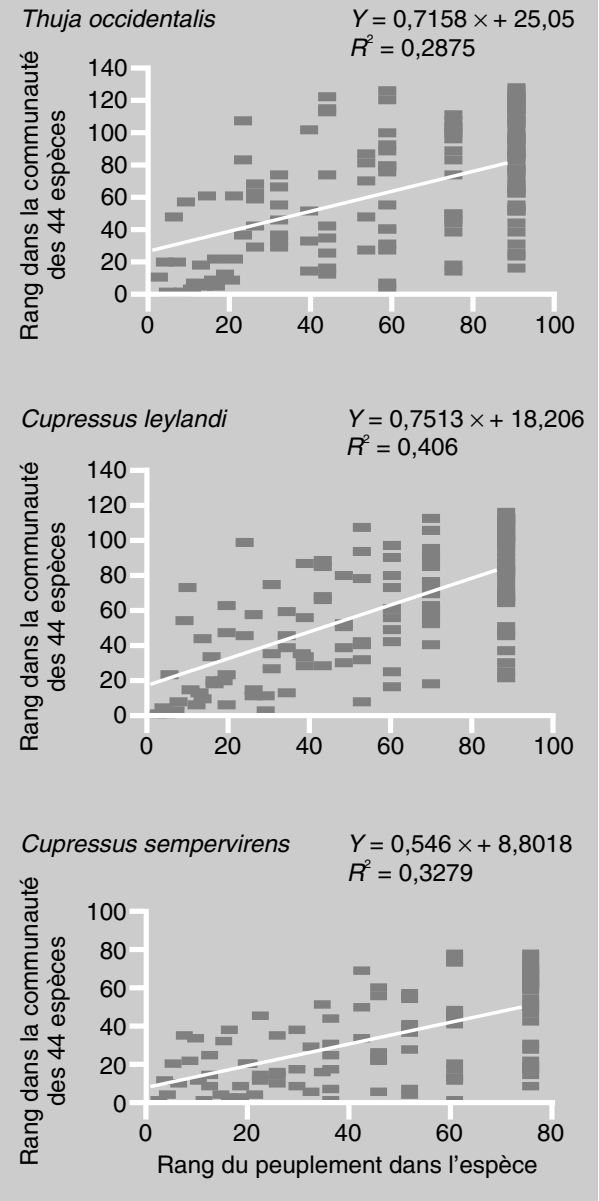

phytophages, les auxiliaires, les autres arboricoles et les recycleurs. La totalité des 746 taxons se sont répartis comme suit : 379 taxons pour les phytophages, 241 taxons pour les auxiliaires - dont 195 taxons pour les prédateurs et 46 taxons pour les parasitoïdes -, 73 taxons pour les recycleurs et 53 taxons pour les autres arboricoles. 


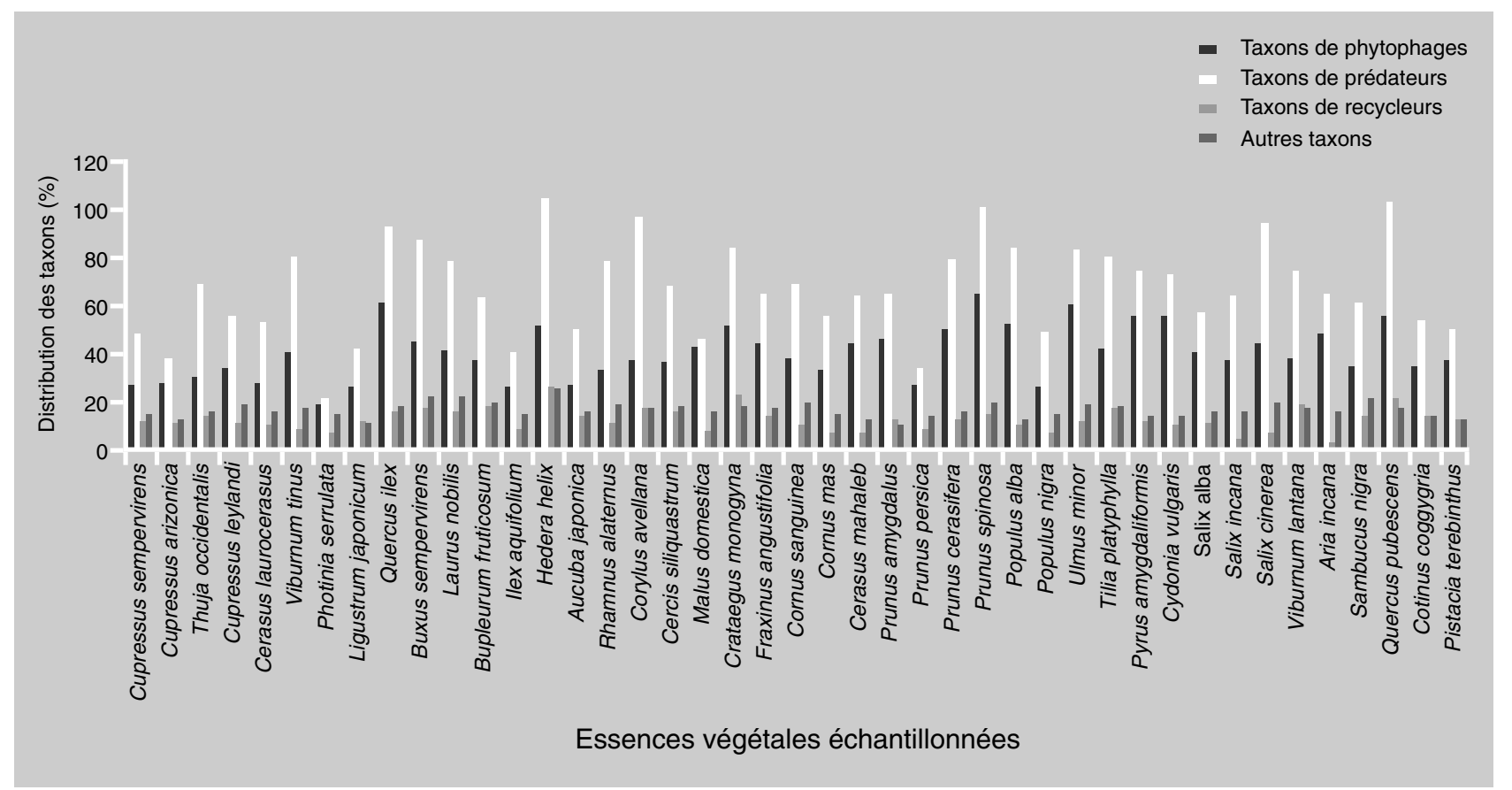

Figure 2.

Biodiversité relative des auxiliaires, des phytophages, des recycleurs et autres éléments arboricoles dans le peuplement des arthropodes de 43 espèces végétales échantillonnées séparément.
La comparaison du nombre de taxons des quatre groupes répertoriés sur les essences végétales considérées une à une a montré que la biodiversité des auxiliaires (prédateurs, parasitoïdes) était supérieure à celle des phytophages (figure 2).

Un végétal unique (biodiversité minimale) offre différents sites qui peuvent être colonisés par des espèces spécifiques de chacun d'eux (racines, tiges, bourgeons, feuilles, fleurs, fruits, diverses catégories de rameaux...). Par exemple, une certaine diversité de phytophages est capable de s'attaquer aux feuilles : thrips, psylles, aleurodes, cochenilles, pucerons, cicadelles, punaises, chenilles, divers coléoptères... Ces phytophages sont à leur tour consommés par un cortège d'entomophages. Ainsi, parmi ces consommateurs de feuilles, les psylles, par exemple, sont victimes d'un assortiment diversifié de parasitö̈des spécifiques et de prédateurs exclusifs ou préférentiels, ainsi que de prédateurs plus généralistes ; pour cette seule catégorie de ravageurs, il est possible de dresser une liste de plusieurs dizaines d'espèces entomophages qui en font leur nourriture exclusive ou favorite.
Ainsi, pour chaque espèce phytophage, la diversité des consommateurs est multipliée de façon considérable. L'étage des entomophages, à rôle régulateur, est considérablement plus diversifié que celui des phytophages. Pour chacune des essences, la biodiversité des auxiliaires est supérieure à celle des phytophages.

En revanche, à l'échelle des 44 essences considérées globalement, c'est la diversité des phytophages qui est supérieure à celle des auxiliaires (figure 3). En effet, si la monospécificité végétale limite davantage les phytophages que les auxiliaires, à l'inverse, la multispécificité privilégie la diversité des phytophages. La situation la plus favorable aux cultures se situe dans une certaine gamme de paucispécificité de leur environnement végétal intentionnel. En fait, dans le cas d'un peuplement végétal diversifié, chaque végétal possède ses phytophages spécifiques mais partage, avec d'autres espèces végétales, une faune plus diversifiée d'espèces entomophages. En raison du faible chevauchement de l'étage des phytophages et du fort chevauchement de celui des entomophages, il est à prévoir que l'assemblage d'un grand nombre d'espèces végétales se soldera par une augmentation 
continue de la diversité des phytophages, et donc du risque de renforcer les ravageurs de la culture, ainsi que par une saturation progressive de l'étage des auxiliaires déjà largement possédés en commun par les espèces végétales précédentes. Pour un cortège végétal diversifié, le panorama sera inverse de celui du végétal unitaire et présentera donc une dominance de la diversité des phytophages par rapport à celle des entomophages. Les observations ont confirmé ces prévisions. Lors de l'aménagement des abords d'un champ, il conviendra donc de ne pas accroître inconsidérément la diversité végétale, ce qui ne conduirait qu'à augmenter le risque dû aux phytophages.

\subsection{Les essences végétales et leur peuplement de phytophages}

Dans la mise en place d'une haie composite ou lors de l'aménagement d'un bord de champ, il sera également important d'éviter les essences végétales dont le peuplement en phytophages se rapprocherait de celui de la culture considérée.

En considérant que les peuplements par essence étaient spécifiques et que la collection des 44 essences échantillonnées au cours de notre étude représentait le meilleur échantillon disponible de la communauté générale de l'environnement, nous avons pu alors confronter la communauté de chaque essence à cette communauté générale en suivant la méthodologie suivante.

- La somme des récoltes effectuées sur ces 44 essences a permis de constituer un échantillon environnemental, ou échantillon global, qui, loin d'être un ensemble amorphe, possédait sa propre structure. Nous avons pu calculer, pour chaque taxon, sa fréquence d'occurrence par essence, depuis les taxons présents sur une seule essence (fréquence $=1 / 44$ ) jusqu'à ceux observés sur toutes les essences (fréquence $=44 / 44)$. Ces fréquences d'occurrence de taxons ont alors été transformées en pourcentage et les effectifs de taxons en logarithme ; les taxons de fréquence supérieure à $50 \%$ et d'effectif inférieur à 3, considérés comme propres à une essence, ont été extraits de l'ensemble et projetés sur un système simple de coordonnées portant en ordonnée leur fréquence dans la communauté générale et en abscisse le logarithme de leur effectif dans la communauté générale. L'analyse de la représentation obtenue par cette méthode a montré que tout se passait comme si, dans ce système de projection, les espèces étaient attirées par deux pôles opposés : celui des espèces ubiquistes, et celui des espèces spécifiques (figure 4). La caractérisation d'un sousnuage des ubiquistes et d'un sous-nuage des spécifiques précédemment exposée a permis d'isoler un noyau d'espèces relativement liées à l'essence considérée.

- La projection de cette séparation sur le plan défini par la fréquence d'occurrence en fonction du logarithme de l'effectif prend la forme d'un nuage présentant une raréfaction des points dans la zone des $50 \%$ d'occurrence. Les espèces constantes sont présentes dans plus de $50 \%$ des relevés, celles "accessoires " sont présentes dans (25 à 50) \% des relevés et enfin les espèces accidentelles sont présentes dans moins de $25 \%$ des relevés.

- Le faible nombre de taxons aux alentours de la fréquence des $50 \%$ semble être une caractéristique générale des communautés. Il semblerait que, sélectivement, les espèces réussissent mieux si elles sont soit spécifiques soit ubiquistes. Ayant ainsi isolé le cortège des taxons spécifiques à chaque essence, nous avons pu tenter des comparaisons entre communautés qui ne portent que sur le cortège des taxons spécifiques, nous avons en quelque sorte éliminé le "bruit de fond " des ubiquistes

Ces comparaisons ont été conduites sur les phytophages qui constituent la fraction la plus dangereuse du cortège, mais qui ne représentent pas l'ensemble de la communauté des phytophages. Elles ont été réalisées à l'aide du coefficient $S$ de Sorensen (tableau I).

Les valeurs de $S$ semblent ranger les essences dans le même ordre que leur degré d'affinité botanique : il est possible de faire ressortir des différents groupes les cortèges des phytophages qui ont une similarité d'autant plus élevée que les végétaux-hôtes présentent une affinité botanique plus étroite. En revanche, les comparaisons

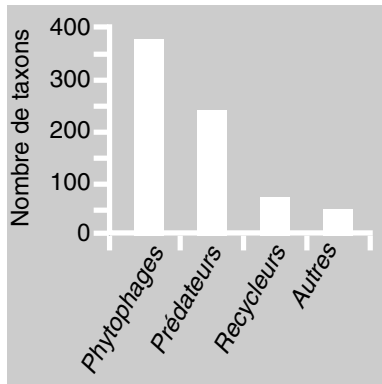

Figure 3.

Biodiversité des auxiliaires, des phytophages, des recycleurs et autres éléments arboricoles dans le peuplement des arthropodes de 43 espèces végétales considérées globalement. 

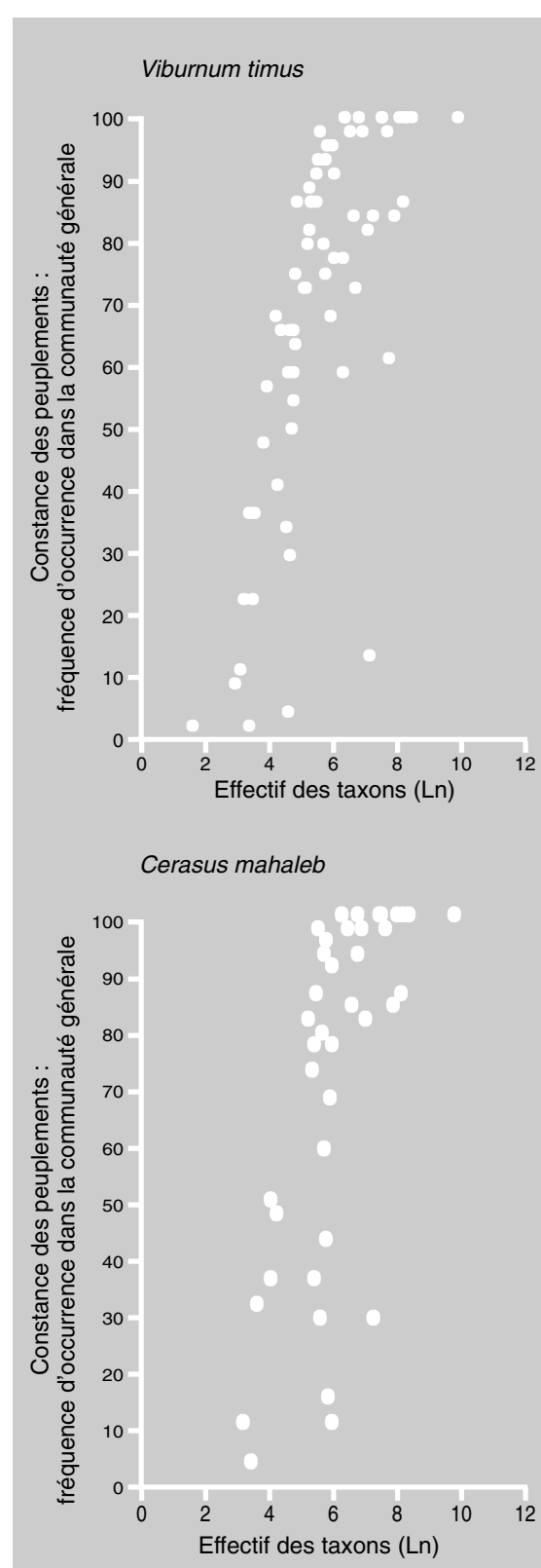

\section{Figure 4.}

Constance des peuplements par essence végétale : les fréquences d'occurrence de taxons dans la communauté globale ont été transformées en pourcentage et les effectifs de taxons en logarithme.
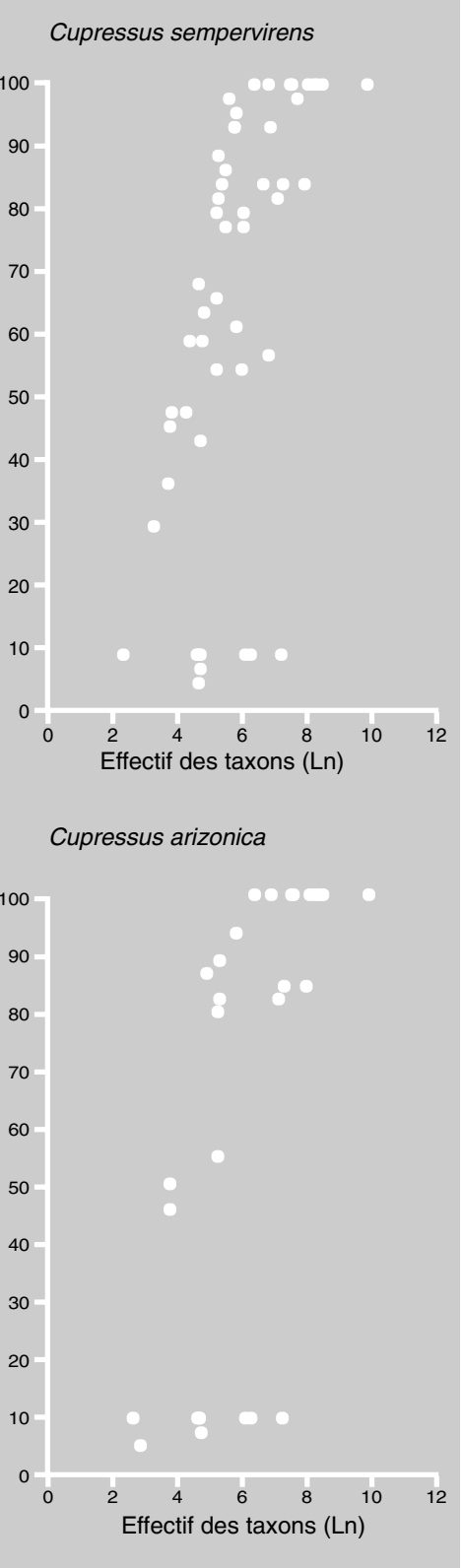

Figure 5.

Regroupement en quinze groupes des taxons d'auxiliaires récoltés sur 43 essences végétales échantillonnées dans des haies aux abords de cultures. menées entre le peuplement des phytophages dits " spécifiques " des 44 essences végétales et le peuplement des phytophages dits "spécifiques " du poirier n'ont pas été significatives ; le peuplement en phytophages des essences végétales considérées ne semblerait donc pas présenter de similitude avec le peuplement en phytophages du poirier.

\subsection{Ressemblance des peuplements d'auxiliaires des essences végétales avec celui du poirier}

Le calcul du coefficient de corrélation de Spearman a permis de mettre en évidence d'éventuelles similitudes de peuplement d'auxiliaires entre les 43 essences végétales échantillonnées et le poirier. Afin que le résultat porte sur la totalité du peuplement des auxiliaires, quinze groupes contenant l'ensemble de la faune auxiliaire ont été définis au préalable (figure 5) et le coefficient a donc été calculé sur ces quinze groupes.

Si, comme nous l'avons vu précédemment, les peuplements en phytophages de l'ensemble des essences étudiées se sont révélés différents de ceux du poirier, les peuplements en auxiliaires apparaissent

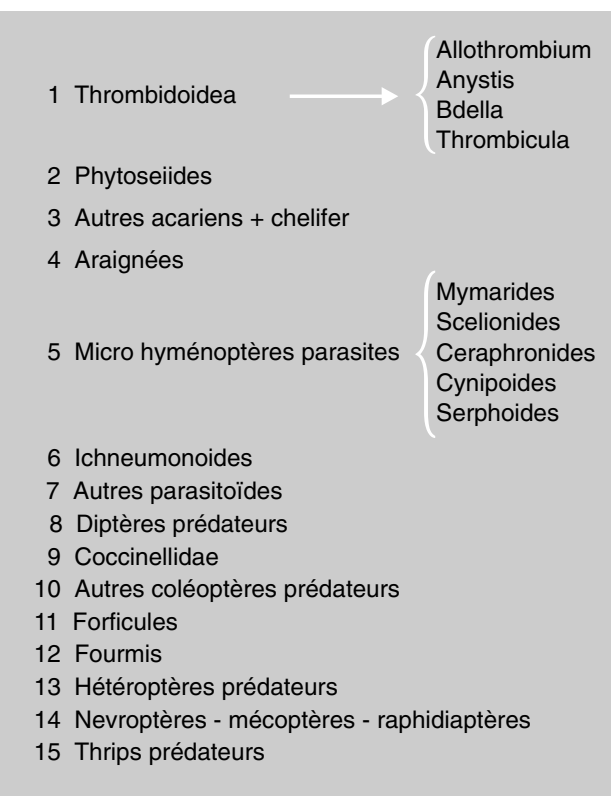


proches pour un certain nombre d'espèces chez lesquelles de coefficient de corrélation de rang de Spearman $r$ a été trouvé supérieur à 0,62 (tableau II).

L'existence de corrélations significatives entre l'entomofaune des auxiliaires de certaines essences végétales et celle du poirier a ainsi été mise en évidence. Le poirier présente ainsi une corrélation significative avec le frêne ainsi qu'avec le lierre. Le frêne, comme le poirier, héberge des psylles, des pucerons et des cécidomyies spécifiques, ce qui peut rendre compte de la ressemblance du cortège de leurs auxiliaires : nous pourrions schématiquement dire que le frêne a une "richesse de garde-manger " alors que le lierre, avec ses feuilles persistantes et ses entrelacements de tiges, a une "richesse d'abri ". Si ces essences exercent une influence sur le poirier, celle-ci sera donc complémentaire. L'arbre de Judée (Cercis siliquastrum, famille des césalpiniées) avec les populations saisonnières de son psylle migrant est également à retenir. Il constitue un garde-manger saisonnier pour les auxiliaires recherchés, qui se vide brutalement fin mai/début juin lors de la migration, contraignant ces auxiliaires à se répandre sur d'autres arbres à psylles pour survivre. Or, cette époque coïncidant avec l'apparition du risque de pullulation du psylle du poirier, l'arbre de Judée réaliserait alors une sorte de "lâcher automatique d'auxiliaires " au moment le plus propice pour la culture.

Pour l'aménagement du bord d'un champ, pourront être ainsi retenus les végétaux hébergeant une ou plusieurs espèces phytophages spécifiques (ne passant donc pas sur les cultures) dont les populations occupent de façon prépondérante la niche écologique que constitue chacune de ces essences. En outre, cette place prise restreint d'autant les risques d'installation de phytophages polyphages auxquels, de toute façon, la culture reste soumise même en l'absence d'environnement intentionnel. Dans le cas du poirier, afin de limiter les risques des principales maladies de celuici, les végétaux seront choisis de préférence parmi des familles botaniquement éloignées (caprifoliacées, rhamnacées, lauracées...) du groupe systématique (ici
Tableau II.

Mesure des ressemblances du cortège des auxiliaires entre diverses essences par le coefficient de corrélation de Spearman de façon à mettre en évidence d'éventuelles similitudes de peuplement entre essences. Pour $n=15$ et à $5 \%$, le seuil est $r_{s}=0,62$.

Essence végétale Corrélation $>0,62$ Corrélation $<0,62$

Arbres de haut jet

Populus nigra

Salix alba

0,879

Ulmus minor

Populus alba

Fraxinus angustifolia

Tilia platyphylla

Salix cinerea

0,793

0,739

0,734

0,730

$-$

$-$

Espèces Intermédiaires

Cupressus sempervirens $\quad 0,902$

Cerasus laurocerasus $\quad 0,852$

Malus communis $\quad 0,800$

Quercus ilex

Cupressus leylandi

Quercus pubescens

Sambucus nigra

Photinia serrulata

Pistacia terebinthus

Aria incana

Prunus persica

Ligustrum japonicum

Cupressus arizonica

Amygdalus communis

Cerasus mahaleb

Espèces intercalaires

Cydonia vulgaris

Laurus nobilis

Crataegus monogyna

Ilex aquifolium

Prunus cerasifera

Cercis siliquastrum

Viburnum tinus

Cotinus coggygria

Viburnum lantana

Pyrus amygdaliformis

Corylus avellana

Salix incana

Thuja occidentalis

Aucuba japonica

Espèces de bourrage

Rhamnus alaternus

Prunus spinosa

Cornus sanguinea

Bupleurum fruticosum

Buxus sempervirens

Cornus mas

$-$

$-$

0,532

$-0,396$

Hedera helix
0,800

0,739

0,700

0,650

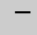

$-$

$-$

$-$

$-$

$-$

$-$

$-$

0,827

0,798

0,789

0,718

0,707

0,680

0,680

0,648

0,629

$-$

-

$-$

$-$

$-$

$-$

$-$

$-$

-

0,525

0,479

0,468

0,425

0,357

0,186

0,168

$-0,057$

0,768

0,709

0,689

0,671

$-$

- $\quad 0,296$
$-$

$-$

$-$

$-$

$-$

$-$

$-$

0,561

0,536

0,063

$-$

$-$

$-$

$-$

$-$ 
rosacées pyrées) auquel appartient la culture à aménager. En outre, certaines essences ne sauraient être retenues malgré un grand intérêt pour leur faune auxiliaire en raison des risques encourus du fait de leur faune de ravageurs et de l'hébergement de diverses maladies. Une grande attention doit être portée à la prise en considération des risques potentiels ou réels que présente chaque espèce végétale vis-à-vis de la culture considérée. Ainsi devront être exclus, en ce qui concerne notre étude :

- l'aubépine, hôte de nombreux auxiliaires en raison de son cortège de psylles spécifiques mais hébergeant, aussi, comme de nombreuses autres rosacées pyrées, l'agent responsable du feu bactérien;

- le prunellier épineux hébergeant à la fois plusieurs maladies à mycoplasmes ou bactéries et leurs insectes vecteurs, cette dernière essence s'avérant par ailleurs difficile à conduire dans une haie en raison d'un drageonnement envahissant.

En fait, la corrélation que nous avons mesurée implique davantage la spécificité des peuplements par essence végétale (mise en évidence plus haut) qu'une quelconque relation de proximité spatiale. Ce résultat montre que, vis-à-vis du poirier, toutes les essences n'ont pas le même intérêt pour leur peuplement des principaux auxiliaires. Il existe des végétaux à forte ou à faible corrélation pour la faune utile. Cette corrélation implique surtout la spécificité du peuplement des essences entre lesquelles elle est mesurée.

\section{Conclusion}

La prise en compte des divers résultats que nous avons exposés montre qu'il n'est pas utopique d'escompter manipuler spécifiquement le cortège des auxiliaires aux abords des cultures [11]. Ce sont eux qui, à l'échelle de l'espèce végétale unitaire, possèdent la biodiversité dominante et il existe une spécificité du peuplement par espèce végétale permettant une utilisation orientée [12] ; il est donc possible de repérer les essences qui ont pour la culture visée le maximum d'intérêt [13]. En outre, si le végétal à implanter est choisi dans une famille botanique à très faible affinité avec l'espèce cultivée, il pourra être tout à fait envisageable de retenir les plantes ayant à la fois une forte corrélation pour les auxiliaires et une très faible corrélation pour les phytophages.

\section{Références}

[1] Ferron P., Bases écologiques de la protection des cultures, gestion des populations et aménagement de leurs habitats, Courr. Environ. Inra 41 (2000) 33-41.

[2] Debras J.F., Cousin M., Rieux R., Conception d'une haie fonctionnelle pour lutter contre le psylle du poirier, Phytoma Déf. Vég. (Fr.) 525 (2000) 44-48.

[3] Perrin R.M., The role of environmental diversity in crop protection, Prot. Ecol. 2 (2) (1980) 77-114.

[4] Baudry O., La haie et la richesse écologique des espaces agricoles. Les brise-vent, réservoirs de faune auxiliaire, Bull. Vulg. For. 52 (1988) 26-27.

[5] Rieux R., Faivre d'Arcier F., Lyoussooufi A., Environnement végétal et auxiliaires entomophages dans le cas des vergers de poiriers, Adalia 2 (1986) 51-52.

[6] Debras J.F., Cousin M., Rieux R., Principes de bases pour la création de haies réservoir d'auxiliaires, Phytoma Déf. Vég. (Fr.) 536 (2001) 26-31.

[7] Legendre L., Legendre P., Écologie numérique : 2 , la structure des données écologiques, Collect. Écol., Masson, Paris, Presses Univ. Québec, Canada, n 13, 1979.

[8] Routledge R.D., Diversity indices: which ones are admissible?, J. Theor. Biol. 76 (1979) 503-515.

[9] Debras J.F., Rieux R., Populations d'arthropodes et itinéraires techniques, Adalia 43 (2000) 2-7.

[10] Fauvel G., Rambier A., Balduque-Martin R., La technique du battage pour la surveillance des ravageurs en culture fruitière et florale. I. Comparaison des résultats obtenus en verger de pommiers avec des entonnoirs rigides de taille moyenne et avec des entonnoirs de toile. Étude de l'influence de quelques facteurs sur l'efficacité du battage, Agronomie 1 (1981) 105-113. 
[11] Ali A.D., Reagan T.E., Vegetation manipulation impact on predator and prey populations in Louisiana sugarcane ecosystems, J. Econ. Entomol. 78 (6) (1985) 1409-1414.

[12] Fye R.E., Cover crop manipulation for building pear psylla (Homoptera: Psyllidae) pred- ator populations in pear orchards, J. Econ. Entomol. 76 (2) (1983) 306-310.

[13] Bernadette L., Intérêt du peuplement en arthropodes de quelques essences de haies composites vis-à-vis de vergers de poiriers conduits en protection intégrée, Isab, mém., Beauvais, France, 1993, 130 p.

\section{Medida de la semejanza de la fauna útil del peral con la de 43 especies vegetales para optimizar la composición de setos reservorios de auxiliares.}

Resumen - Introducción. Dentro del establecimiento de medidas que hay que promover en el marco de una protección integrada, el ordenamiento de las inmediaciones de los cultivos constituye un objetivo principal. Sin embargo, hasta ahora, esta ordenación se ha realizado sin un verdadero criterio. El presente estudio muestra cómo es posible razonar la elección de las especies. Material y métodos. Para cada una de estas especies, y a razón de 30 árboles por mes durante 10 meses del año 1998, se observó la entomofauna de 44 especies leñosas y, entre ellas, la del peral cultivado. La recolección de la entomofauna se efectuó mediante el método de batido de Fauvel. Resultados y discusión. Se efectuaron investigaciones fundamentales sobre la composición y la estructura de las comunidades animales que albergan las frondosas y se compararon con las de un huerto de perales. Se estudió así la fauna de 43 especies leñosas. Tras mostrar la especificidad de las poblaciones, se evidenciaron correlaciones de rango de Spearman entre la población de auxiliares de las 43 especies y el del peral mostrando una semejanza entre la entomofauna útil del peral y la de ciertas especies vegetales. Conclusión. Los resultados obtenidos permiten razonar la elección de especies que pueden servir de reservorio a una entomofauna predadora dentro del marco de la protección integrada del huerto de perales. Este método es aplicable a otros tipos de cultivo.

Francia / Pyrus communis / control de insectos / lucha integrada / plantas para cercas vivas / artrópodos para control biológico / medio ambiente

To access this journal online: www.edpsciences.org 
